\title{
Magnetic and electric screening masses from Polyakov-line correlations
}

\author{
Yu Maezawa ${ }^{* a}$, S. Aoki ${ }^{b, c}$, S. Ejiri ${ }^{d}$, T. Hatsuda ${ }^{e}$, N. Ishii ${ }^{f}$, K. Kanaya ${ }^{b}$, N. Ukita ${ }^{f}$, and \\ T. Umeda ${ }^{b}$ (WHOT-QCD Collaboration) \\ ${ }^{a}$ En'yo Radiation Laboratory, Nishina Accelerator Research Center, RIKEN, Wako, Saitama \\ 351-0198, Japan \\ ${ }^{b}$ Graduate School of Pure and Applied Sciences, University of Tsukuba, Tsukuba, Ibaraki \\ 305-8571, Japan \\ ${ }^{c}$ RIKEN BNL Research Center, Brookhaven National Laboratory, Upton, NY 11973, USA \\ ${ }^{d}$ Physics Department, Brookhaven National Laboratory, Upton, New York 11973, USA \\ ${ }^{e}$ Department of Physics, The University of Tokyo, Tokyo 113-0033, Japan \\ ${ }^{f}$ Center for Computational Sciences, University of Tsukuba, Tsukuba, Ibaraki 305-8577, Japan \\ E-mail: maezawa@ribf.riken.jp
}

\begin{abstract}
Screening properties of the quark gluon plasma are studied from the Polyakov-line correlations in lattice QCD simulations with two flavors of improved Wilson quarks at temperatures $T / T_{\mathrm{pc}} \simeq 1-4$ where $T_{\mathrm{pc}}$ is the pseudocritical temperature. Using the Euclidean-time reflection symmetry and the charge conjugation symmetry, we introduce various types of Polyakov-line correlations and extract screening masses in magnetic and electric sectors. We find that a ratio of the screening masses in the electric sector to the magnetic sector shows qualitative agreement with a prediction from the dimensionally-reduced effective field theory and the $\mathscr{N}=4$ supersymmetric Yang-Mills theory at $1.3<T / T_{\mathrm{pc}}<3$.
\end{abstract}

The XXVI International Symposium on Lattice Field Theory

July 14 - 19, 2008

Williamsburg, Virginia, USA

\footnotetext{
* Speaker.
} 


\section{Introduction}

Electric and magnetic screening masses of the gluon are the fundamental quantities of the quark-gluon plasma at high temperature [1]. In this report, we employ lattice QCD simulations with two flavors of improved Wilson quarks at temperatures $T / T_{\mathrm{pc}} \simeq 1-4$ and try to extract both screening masses from the correlations of the Polyakov-line operator classified under the the Euclideantime reflection and the charge conjugation [2]. Also, we make a comparison of our results with the predictions from the dimensionally-reduced effective field theory and the $\mathscr{N}=4$ supersymmetric Yang-Mills theory.

\section{Correlations of the Polyakov-line operator}

In order to extract the electric and magnetic screening masses, we classify the Polyakov-line operator into different classes on the basis of the symmetries under the Euclidean time-reflection [2] $(\mathscr{T})$ and the charge conjugation $(C)$. Both are good symmetries of QCD at zero chemical potential. (Note that $\mathscr{T}$ corresponds to the product of the time reversal $T$ and charge conjugation $C$ in the Minkowski space.) Under $\mathscr{T}$, the gluon fields are transformed as,

$$
\vec{A}(\tau, \mathbf{x}) \rightarrow \vec{A}(-\tau, \mathbf{x}), \quad A_{4}(\tau, \mathbf{x}) \rightarrow-A_{4}(-\tau, \mathbf{x}) .
$$

An operator belongs to the magnetic (electric) sector if it is even (odd) under $\mathscr{T}$. On the other hand, the charge conjugation changes the gluon field as,

$$
A_{\mu}(\tau, \mathbf{x}) \rightarrow-A_{\mu}^{*}(\tau, \mathbf{x}) .
$$

The Polyakov-line operator

$$
\Omega(\mathbf{x})=P \exp \left[i g \int_{0}^{1 / T} d \tau A_{4}(\tau, \mathbf{x})\right]
$$

transforms under $\mathscr{T}$ symmetry and $C$ symmetry as

$$
\mathscr{T}: \Omega \rightarrow \Omega^{\dagger}, \quad C: \Omega \rightarrow \Omega^{*} .
$$

Therefore, we define $\mathscr{T}$-even (odd) operator by its Hermitian (anti-Hermitian) component:

$$
\Omega_{\mathrm{M}} \equiv \frac{1}{2}\left(\Omega+\Omega^{\dagger}\right), \quad \Omega_{\mathrm{E}} \equiv \frac{1}{2}\left(\Omega-\Omega^{\dagger}\right),
$$

where the subscript implies that the operator is in the magnetic (electric) sector. Furthermore, they can be decomposed into $C$-even and $C$-odd operators as

$$
\Omega_{\mathrm{M}, \pm}=\frac{1}{2}\left(\Omega_{\mathrm{M}} \pm \Omega_{\mathrm{M}}^{*}\right), \Omega_{\mathrm{E}, \pm}=\frac{1}{2}\left(\Omega_{\mathrm{E}} \pm \Omega_{\mathrm{E}}^{*}\right) .
$$

We can define two types of gauge invariant correlators using Eq.(2.6) as

$$
\begin{aligned}
C_{\mathrm{M},+}^{\mathrm{I}}(r, T) & \equiv\left\langle\operatorname{Tr} \Omega_{\mathrm{M},+}(\mathbf{x}) \operatorname{Tr} \Omega_{\mathrm{M},+}(\mathbf{y})\right\rangle-\langle\operatorname{Tr} \Omega\rangle^{2}, \\
C_{\mathrm{E},-}^{\mathrm{I}}(r, T) & \equiv\left\langle\operatorname{Tr} \Omega_{\mathrm{E},-}(\mathbf{x}) \operatorname{Tr} \Omega_{\mathrm{E},-}(\mathbf{y})\right\rangle,
\end{aligned}
$$


where $r \equiv|\mathbf{x}-\mathbf{y}|$, and the superscript "I" implies that the correlators are gauge invariant. Since $\operatorname{Tr} \Omega_{\mathrm{M},-}=\operatorname{Tr} \Omega_{\mathrm{E},+}=0$, the correlators of these operators are identically zero. If we are allowed to fix gauge, we can construct four types of correlators:

$$
\begin{aligned}
C_{\mathrm{M},+}^{\mathrm{F}}(r, T) & \equiv\left\langle\operatorname{Tr} \Omega_{\mathrm{M},+}(\mathbf{x}) \Omega_{\mathrm{M},+}(\mathbf{y})\right\rangle-\langle\operatorname{Tr} \Omega\rangle^{2}, \\
C_{\mathrm{M},-}^{\mathrm{F}}(r, T) & \equiv\left\langle\operatorname{Tr} \Omega_{\mathrm{M},-}(\mathbf{x}) \Omega_{\mathrm{M},-}(\mathbf{y})\right\rangle \\
C_{\mathrm{E},+}^{\mathrm{F}}(r, T) & \equiv\left\langle\operatorname{Tr} \Omega_{\mathrm{E},+}(\mathbf{x}) \Omega_{\mathrm{E},+}(\mathbf{y})\right\rangle \\
C_{\mathrm{E},-}^{\mathrm{F}}(r, T) & \equiv\left\langle\operatorname{Tr} \Omega_{\mathrm{E},-}(\mathbf{x}) \Omega_{\mathrm{E},-}(\mathbf{y})\right\rangle .
\end{aligned}
$$

where the superscript "F" implies gauge-fixed correlators. In this study, we adopt the Coulomb gauge fixing.

We define the screening masses of these correlators through the fit with the following screened Coulomb form at long distances:

$$
C_{\mathscr{T}, C}^{\mathrm{I}(\mathrm{F})}(r, T) \rightarrow \alpha_{\mathscr{T}, C}^{\mathrm{I}(\mathrm{F})}(T) \frac{e^{-m_{\mathscr{T}, C}^{\mathrm{I}(\mathrm{F})}(T) r}}{r}
$$

where $\alpha_{\mathscr{T}, C}^{\mathrm{I}(\mathrm{F})}(T)$ and $m_{\mathscr{T}, C}^{\mathrm{I}(\mathrm{F})}(T)$ are fitting parameters. Then we can extract six screening masses all together,

$$
\begin{aligned}
m_{\mathscr{T}, C}^{\mathrm{I}(\mathrm{F})}(\text { Magnetic sector }): & m_{\mathrm{M},+}^{\mathrm{I}}, m_{\mathrm{M},+}^{\mathrm{F}}, m_{\mathrm{M},-}^{\mathrm{F}}, \\
m_{\mathscr{T}, C}^{\mathrm{I}(\mathrm{F})}(\text { Electric sector }): & m_{\mathrm{E},-}^{\mathrm{I}}, m_{\mathrm{E},+}^{\mathrm{F}}, m_{\mathrm{E},-}^{\mathrm{F}} .
\end{aligned}
$$

\section{Numerical simulations}

We employ a renormalization group improved gauge action and a clover improved Wilson quark action with two flavors. The simulations are performed on a lattice with a size of $N_{s}^{3} \times N_{t}=$ $16^{3} \times 4$ along lines of constant physics, i.e. lines of constant $m_{\mathrm{PS}} / m_{\mathrm{V}}$ (the ratio of pseudoscalar and vector meson masses) at $T=0$ in the space of simulation parameters. Details of the lines of constant physics with the same actions are summarized in Refs. [3, 4, 5]. We take two values of $m_{\mathrm{PS}} / m_{\mathrm{V}}=0.65$ and 0.80 with the temperature range of $T / T_{\mathrm{pc}} \sim 1.0-4.0$ (9 points) and 1.0-3.0 (7 points), respectively, where $T_{\mathrm{pc}}$ is the pseudocritical temperature along the line of constant physics. The number of trajectories for each run after thermalization is 5000-6000, and we measure physical quantities at every 10 trajectories. The fits are performed by minimizing $\chi^{2} / N_{\mathrm{DF}}$ with fit ranges of $0.35 \leq r T \leq 1.0$ for $C_{\mathrm{E},-}^{\mathrm{I}}$ and $0.5 \leq r T \leq 1.25$ for other orrelators.

Left (right) panel of Fig. 1 shows results of screening masses in magnetic (electric) sector at $m_{\mathrm{PS}} / m_{\mathrm{V}}=0.65$ as a function of temperature. As temperature increases, the magnetic screening masses converge to a similar value, whereas the electric screening masses shows $m_{\mathrm{E},+}^{\mathrm{F}} \simeq m_{\mathrm{E},-}^{\mathrm{F}}<$ $m_{\mathrm{E},-}^{\mathrm{I}}$. In the weak coupling limit, these properties may be interpreted as follows. The Polyakov-line operator in the magnetic (electric) sector has even (odd) powers of $g$ :

$$
\begin{aligned}
& \Omega_{\mathrm{M}} \sim 1+\frac{1}{2}\left(i g \int_{0}^{1 / T} d \tau A_{4}\right)^{2}+\cdots \\
& \Omega_{\mathrm{E}} \sim-i g \int_{0}^{1 / T} d \tau A_{4}-\frac{1}{3 !}\left(i g \int_{0}^{1 / T} d \tau A_{4}\right)^{3}+\cdots .
\end{aligned}
$$



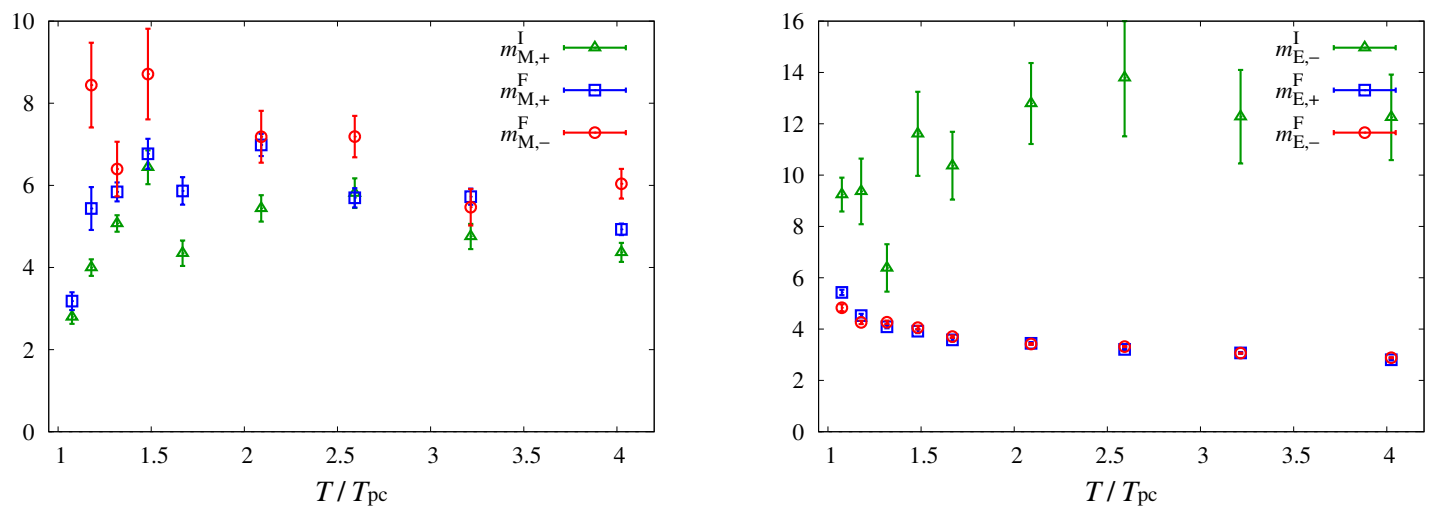

Figure 1: Screening masses in magnetic (left) and electric (right) sectors at $m_{\mathrm{PS}} / m_{\mathrm{V}}=0.65$.

Then all the Polyakov-line correlations in the magnetic sector start with the correlation of the second powers of $A_{4}$ :

$$
\begin{aligned}
& C_{\mathrm{M}}^{\mathrm{F}}(r, T) \propto g^{4}\left\langle\operatorname{Tr} A_{4}^{2}(\mathbf{x}) A_{4}^{2}(\mathbf{y})\right\rangle+O\left(g^{8}\right), \\
& C_{\mathrm{M}}^{\mathrm{I}}(r, T) \propto g^{4}\left\langle\operatorname{Tr} A_{4}^{2}(\mathbf{x}) \operatorname{Tr} A_{4}^{2}(\mathbf{y})\right\rangle+O\left(g^{8}\right) .
\end{aligned}
$$

On the other hand, Polyakov-line correlations in the electric sector have different powers depending on the gauge choice, because $\operatorname{Tr} A_{4}=0$ :

$$
\begin{aligned}
& C_{\mathrm{E}}^{\mathrm{F}}(r, T) \propto g^{2}\left\langle\operatorname{Tr} A_{4}(\mathbf{x}) A_{4}(\mathbf{y})\right\rangle+O\left(g^{6}\right), \\
& C_{\mathrm{E}}^{\mathrm{I}}(r, T) \propto g^{6}\left\langle\operatorname{Tr} A_{4}^{3}(\mathbf{x}) \operatorname{Tr} A_{4}^{3}(\mathbf{y})\right\rangle+O\left(g^{8}\right) .
\end{aligned}
$$

Then the leading contribution in $C_{\mathrm{E}}^{\mathrm{F}}$ comes from single temporal-gluon exchange, whereas that in $C_{\mathrm{E}}^{\mathrm{I}}$ comes from triple temporal-gluon exchange. This may lead to the exponential fall of $C_{\mathrm{E},-}^{\mathrm{I}}$ much faster than $C_{\mathrm{E}, \pm}^{\mathrm{F}}$ and hence the mass ordering in Fig.1. Further discussions on a relation between the Polyakov-line correlations and the gluon propagators in the weak coupling are given in Appendix.

\subsection{Comparison with 3D effective field theory and $\mathscr{N}=4$ supersymmetric Yang-Mills theory}

Let us compare our results with the predictions by the dimensionally reduced effective field theory (3D-EFT) and the $\mathscr{N}=4$ supersymmetric Yang-Mills theory (SYM). Here we focus our attention on the screening masses with $(\mathscr{T}, C)=(+,+)$ and $(-,-)$, i.e. $m_{\mathrm{M},+}$ and $m_{\mathrm{E},-}$, which are channels calculable from the gauge invariant Polyakov-line correlations ${ }^{1}$. In the 3D-EFT approach [6], the screening masses at $T=2 \Lambda_{\overline{M S}}$ with $\Lambda_{\overline{M S}}$ being the QCD scale parameter have been calculated for $N_{f}=2$ as

$$
\text { 3D-EFT }\left(N_{f}=2\right): m_{\mathrm{M},+} \sim 3.60 T, m_{\mathrm{E},-} \sim 6.46 T .
$$

\footnotetext{
${ }^{1}$ Although our Polyakov-line operators do not have definite angular momentum $J$ and the parity $P$, we assume that the asymptotic behavior of our Polyakov-line correlations picks up the ground state contribution corresponding to $(J, P)=(0,+)$
} 


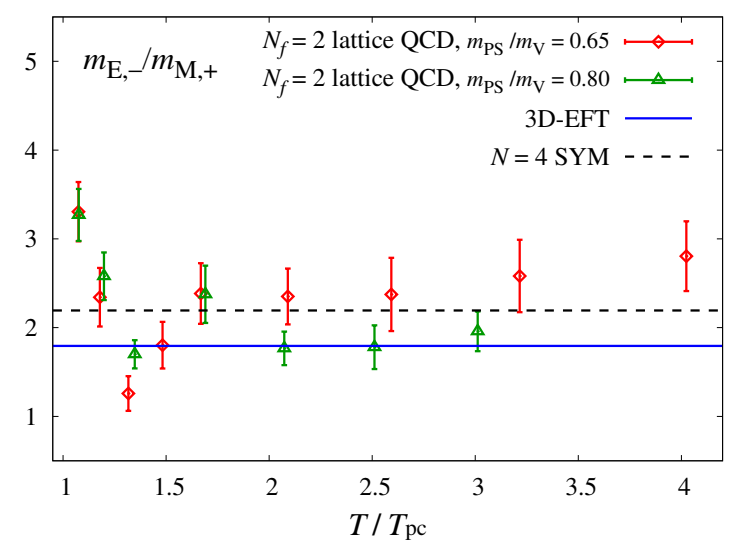

Figure 2: Comparison of the screening ratio, $m_{\mathrm{E},-} / m_{\mathrm{M},+}$, with predictions in the dimensionally-reduced effective field theory (3D-EFT) [6] and $\mathscr{N}=4$ supersymmetric Yang-Mills theory (SYM) [7].

The screening masses of the Polyakov-line correlations in $\mathscr{N}=4$ supersymmetric Yang-Mills theory in the limit of large $N_{c}$ and large 'tHooft coupling $\left(\lambda=g^{2} N_{c}\right)$ were calculated by AdS/CFT correspondence [7]:

$$
\mathscr{N}=4 \mathrm{SYM}: m_{\mathrm{M},+} \sim 7.34 T, m_{\mathrm{E},-} \sim 16.1 T .
$$

Our results of the screening masses shown in Fig. 1 indicates that

$$
\text { 4D-lattice QCD }\left(N_{f}=2\right): m_{\mathrm{M},+}=4 T-6 T, m_{\mathrm{E},-}=10 T-14 T \text {. }
$$

In all three cases, we have the inequality: $m_{\mathrm{M},+}<m_{\mathrm{E},-}$. Since we cannot compare the absolute magnitude of the screening masses in QCD and those in $\mathscr{N}=4$ SYM because of the different number of degrees of freedom, we take the ratio, $m_{\mathrm{E},-} / m_{\mathrm{M},+}$ in Fig. 2 for above three cases. We find that, in spite of the difference in the magnitude, the screening ratios agree well with each other for $1.5<T / T_{\mathrm{pc}}<3$.

\section{Summary}

We investigated the screening masses in hot quark-gluon plasma from the Polyakov-line correlations classified by the Euclidean-time reflection $(\mathscr{T})$ symmetry and the charge conjugation $(C)$ symmetry. The screening masses are calculated in the magnetic ( $\mathscr{T}$-even) and electric ( $\mathscr{T}$-odd) sectors from the lattice simulations of $N_{f}=2 \mathrm{QCD}$ above $T_{\mathrm{pc}}$ with the RG-improved gluon action and the clover-improved Wilson quark action on a $16^{3} \times 4$ lattice. We found that, the magnetic screening masses obtained by the gauge-invariant and gauge-fixed correlations coincide with each other for $T / T_{\mathrm{pc}}>2$, whereas the electric screening mass obtained from the gauge invariant correlator is larger than that obtained from the gauge fixed correlator. Our results obtained from the gauge invariant correlators are compared with the calculations in the dimensionally-reduced effective field theory [6] and the $\mathscr{N}=4$ supersymmetric Yang-Mills theory [7]. We found that the ratio of the electric and magnetic screening masses in three cases are qualitatively consistent with each other for at $1.3<T / T_{\mathrm{pc}}<3$. Further discussions between the Polyakov-line correlations and the gluon propagators are given in Appendix. 
Acknowledgements: We would like to thank M. Laine for giving useful advice about the Euclidean time symmetry. This work is in part supported by Grants-in-Aid of the Japanese Ministry of Education, Culture, Sports, Science and Technology (Nos. 17340066, 18540253, 19549001, and 20340047). SE is supported by U.S. Department of Energy (DE-AC02-98CH10886). Numerical calculations were performed on supercomputers at KEK by the Large Scale Simulation Program Nos. 06-19, 07-18, 08-10, at CCS, Univ. of Tsukuba, and at ACCC, Univ. of Tsukuba.

\section{A. Appendix: Relation to gluon propagators}

In this Appendix, we consider a direct relation between the Polyakov-line correlations and the screening mass in gluon propagators. Here we define the magnetic (electric) screening mass, $m_{M}$ $\left(m_{E}\right)$, from the spatial (temporal) gluon propagator at asymptotic spatial distance,

$$
\begin{aligned}
\left\langle A_{i}(\mathbf{x}) A_{i}(\mathbf{y})\right\rangle \rightarrow T \frac{e^{-m_{M} r}}{4 \pi r} \quad(|\mathbf{x}-\mathbf{y}|=r \rightarrow \infty), \\
\left\langle A_{4}(\mathbf{x}) A_{4}(\mathbf{y})\right\rangle \rightarrow T \frac{e^{-m_{E} r}}{4 \pi r} \quad(|\mathbf{x}-\mathbf{y}|=r \rightarrow \infty) .
\end{aligned}
$$

In the weak coupling limit, the gauge fixed correlation $C_{\mathrm{E},-}^{\mathrm{F}}$ is related to the electric propagator as

$$
C_{\mathrm{E},-}^{\mathrm{F}}(r, T) \rightarrow a(T) \frac{e^{-m_{E}(T) r}}{r T} .
$$

On the other hand, the gauge invariant correlation $C_{\mathrm{M},+}^{\mathrm{I}}$ would have not only an exchange of two temporal gluons but also an exchange of two spatial gluons [2]:

$$
C_{\mathrm{M},+}^{\mathrm{I}}(r, T) \rightarrow b_{1}(T)\left(\frac{e^{-m_{E}(T) r}}{r T}\right)^{2}+b_{2}(T)\left(\frac{e^{-m_{M}(T) r}}{r T}\right)^{2} .
$$

Although $b_{2}$ has higher powers in $g$, the exchange of the spatial gluons could dominate at long distances if $m_{M}<m_{E}$.

We extract $m_{E}$ by fitting $C_{\mathrm{E},-}^{\mathrm{F}}$ to the right-hand-side of Eq. (A.3) with the fitting parameters, $a(T)$ and $m_{E}(T)$. Then we extract $m_{M}$ by fitting a combination of the $C_{\mathrm{E},-}^{\mathrm{F}}$ and $C_{\mathrm{M},+}^{\mathrm{I}}$ as,

$$
\frac{C_{\mathrm{M},+}^{\mathrm{I}}(r, T)}{\left(C_{\mathrm{E},-}^{\mathrm{F}}(r, T)\right)^{2}}=c_{1}+c_{2}(T) \exp \left[2\left(m_{E}(T)-m_{M}(T)\right) r\right],
$$

with $c_{1}, c_{2}$ and $m_{M}$ being the fitting parameters. The fit ranges are chosen to be $0.5 \leq r T \leq 1.25$.

Results of $m_{E}(T)$ and $m_{M}(T)$ are shown in Fig. 3 (left) as a function of $T / T_{\mathrm{pc}}$ at $m_{\mathrm{PS}} / m_{\mathrm{V}}=$ 0.65. Similar results are obtained for $m_{\mathrm{PS}} / m_{\mathrm{V}}=0.80$ too. We find that $m_{M}$ is smaller than $m_{E}$ at all temperatures we calculate, which is consistent with a prediction in the thermal perturbation theory: $m_{M} \sim O\left(g^{2} T\right)<m_{E} \sim O(g T)$. We also find that, for $T / T_{\mathrm{pc}}>2$, both screening masses decreases as $T$ increases, whereas $m_{E}$ and $m_{M}$ behaves differently for $1<T / T_{\mathrm{pc}}<2$, i.e. $m_{E}$ increases and $m_{M}$ decreases when the temperature approaches to $T_{\mathrm{pc}}$. The behavior of $m_{M}$ is not expected from the leading-order perturbation theory.

In the right panel of Fig. 3, $m_{E}$ and $m_{M}$ calculated directly from the gluon propagators in the quenched approximation are recapitulated [8]. Although $m_{M}<m_{E}$ is also found for $T / T_{\mathrm{c}} \gtrsim 1.2$, 

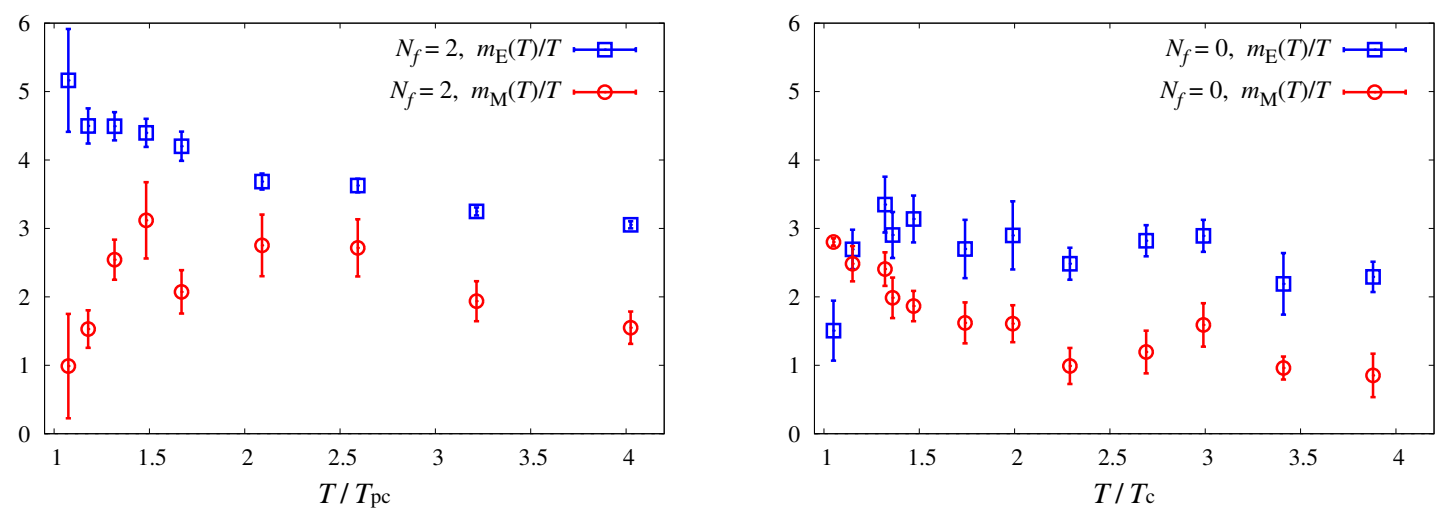

Figure 3: Results of the electric and magnetic screening masses calculated from the Polyakov-line correlations in $N_{f}=2$ lattice QCD at $m_{\mathrm{PS}} / m_{\mathrm{V}}=0.65$ (left) and from the gluon propagators in $N_{f}=0$ lattice QCD [8] (right).

$m_{M}\left(m_{E}\right)$ increases (decreases) as $T$ approaches to $T_{\mathrm{c}}$ in the quenched calculation. Such qualitative difference between $N_{f}=2$ and $N_{f}=0$ near the (pseudo-) critical temperature may be attributed to the different behavior of the the phase transition in two cases.

\section{References}

[1] U. Kraemmer and A. Rebhan, Rept. Prog. Phys. 67, 351 (2004).

[2] P. Arnold and L. G. Yaffe, Phys. Rev. D 52, 7208 (1995).

[3] A. Ali Khan et al. (CP-PACS Collaboration), Phys. Rev. D 63034502 (2001).

[4] A. Ali Khan et al. (CP-PACS Collaboration), Phys. Rev. D 64074510 (2001).

[5] Y. Maezawa et al. (WHOT-QCD Collaboration), Phys. Rev. D 75, 074501 (2007).

[6] A. Hart, M. Laine and O. Philipsen, Nucl. Phys. B 586, 443 (2000).

[7] D. Bak, A. Karch and L. G. Yaffe, JHEP 0708, 049 (2007).

[8] A. Nakamura, T. Saito and S. Sakai, Phys. Rev. D 69, 014506 (2004). 